\title{
EXPERIENCIA DE LA ENFERMEDAD HIPERTENSIVA Y ADHERENCIA EN PERSONAS HIPERTENSAS PERTENECIENTES AL PUEBLO ORIGINARIO MAPUCHE
}

\author{
EXPERIENCE OF HYPERTENSIVE DISEASE AND TREATMENT \\ ADHERENCE IN ETHNIC MAPUCHE HYPERTENSIVE PATIENTS
}

\author{
Alejandro Ramírez Jaramillo* \\ GABRIELA NAZAR ${ }^{* *}$
}

\begin{abstract}
RESUMEN
Objetivo: Comprender la experiencia de la enfermedad hipertensiva y su tratamiento, y los facilitadores y obstaculizadores de la adherencia en personas mapuche con hipertensión. Método: Cualitativo descriptivo de enfoque fenomenológico, con muestreo intencionado y análisis temático de entrevistas semiestructuradas a 12 participantes hipertensos de la etnia mapuche pertenecientes a un programa cardiovascular en una zona rural del centro sur en Chile. Resultados: La hipertensión se vivencia con desconocimiento, en referencia al sistema occidental de salud y centrada en síntomas fisiológicos como dolor de cabeza y mareos. El tratamiento se percibe con desconfianza y la calidad de la atención deficiente. La familia destaca como mediadora del tratamiento en adherentes y ausente en los no adherentes. Se ingiere preparaciones de plantas naturales para potenciar el efecto del fármaco. No hay referencia a enfermedades y espiritualidad mapuche, y la consulta a agentes ancestrales de salud fue escasa. Conclusión: El conocimiento insuficiente de la hipertensión, desconfianza con el tratamiento, insatisfacción con el trato y el complemento o reemplazo del fármaco por preparados de plantas naturales dificulta la adherencia al tratamiento en indígenas mapuche. La ausencia de referentes sanadores ancestrales, de las categorías mapuche de enfermedad y de la espiritualidad, puede deberse al alejamiento de las tradiciones mapuche. La definición del problema y su tratamiento desde parámetros occidentales indicaría que la tensión cultural está sobredimensionada para explicar la no adherencia.
\end{abstract}

Palabras clave: Adherencia terapéutica, hipertensión, grupos étnicos.

\begin{abstract}
Objective: To describe and understand the experience of hypertension as well as factors that help or hinder treatment adherence in Mapuche patients enrolled in a cardiovascular program in the Arauco health service. Method: Qualitative and descriptive study with a phenomenological focus. Sample was comprised of twelve hypertensive Mapuche patients who were subjects of an in-depth interview. Thematic analysis was carried out for information analysis. Results: The hypertension was experienced with unfamiliarity and in reference to the western health system, focused mainly on physiological symptoms such as headaches and dizziness. Treatment was perceived with distrust and the quality of care was perceived as poor. Family stands-out as a mediator of treatment in adherent patients and absent in non-adherents, and a source of information about medicinal herbs. Patients drink herbal infusions to maximize the effects of prescribed medications. There was no reference to any Mapuche disease category or spirituality, and patients did not seek help from health ancestral

\footnotetext{
•Psicólogo Unidad Salud Intercultural, Servicio de Salud Arauco. Carrera 302, Lebu, Chile. Email: jaramillo.ramirez@ gmail.com

"Psicóloga. Departamento de Psicología. Centro de Vida Saludable. Universidad de Concepción. Casilla 160 C. Concepción, Chile. Email: gnazar@udec.cl. Autor de correspondencia.
} 
agents. Conclusion: Insufficient knowledge of hypertension, lack of trust in the treatment, the replacement of prescribed medication with medicinal herbs are some of the obstacles in adherence to treatment in Mapuche patients. Not consulting health ancestral agents and a lack of reference to any Mapuche disease category and spirituality, may be due to the loss of Mapuche traditions. The definition of the health problem and its treatment from a Western perspective would be showing that the cultural tension is over emphasized and explain lack of treatment adherence.

Key words: Treatment adherence and compliance, hypertension, ethnic groups.

Fecha recepción: 17/01/17 Fecha aceptación: 09/04/18

\section{INTRODUCCIÓN}

Las enfermedades cardiovasculares representan un problema de salud pública prioritario para los países industrializados y en desarrollo, por su alto impacto sanitario y económico, siendo la principal causa de morbimortalidad en el adulto $(1,2)$. De ellas, la hipertensión arterial es una de las patologías más frecuentes, estimándose una prevalencia mundial entre el 20 y $26 \%$ y su condición como uno de los mayores factores de riesgo de morbimortalidad cardiovascular y cerebrovascular en el mundo (2). No obstante su prevalencia, el control de la enfermedad ha resultado insuficiente, generando bajos resultados en salud y elevados costos sanitarios. Por tanto incentivar la adherencia tendría una mayor repercusión en el control de esta patología, que si solamente se mejoran los tratamientos médicos (3).

La Encuesta Nacional de Salud 2009-2010 (4) señala que las enfermedades cardiovasculares representan la principal carga de salud del país, y se asocian a hábitos adquiridos en la infancia o adolescencia relacionados con una múltiple red de determinantes sociales responsables de las inequidades en salud. Algunos de ellos socio-individuales como las prácticas en salud, y otros estructurales como la pobreza, la educación y la etnia (5).

Estas diferencias culturales en la comprensión y manejo de los temas relacionados con la salud están a la base de los perfiles de mayor riesgo y sobremortalidad que demuestran estudios epidemiológicos en grupos étnicos chilenos (6-9).

La adherencia al tratamiento ha sido definida como una alianza terapéutica con la implicación activa y voluntaria del paciente, en un curso de comportamiento aceptado de mutuo acuerdo para producir un resultado deseado $(10,11)$. El paciente adhiere a un plan que acepta y que ha acordado con el médico tratante y en el cual se supone ha podido participar en su elaboración $(10,11)$.

La adherencia al tratamiento se describe como un comportamiento complejo influido por múltiples factores que se pueden clasificar en: socioeconómicos, de la atención médica, de la enfermedad, el tratamiento y factores del paciente. Entre estos últimos se identifican las creencias socioculturales o étnicas relacionadas con la enfermedad y su tratamiento $(12,13)$.

Los aspectos psicológicos, emocionales y de relación también se reconocen como involucrados directamente con la adherencia $(14,15)$. El trato individualizado, la comunicación y el apoyo social facilitarían la generación de lazos afectivos y la autoeficacia percibida para el autocuidado (16).

Para la comprensión de la enfermedad en su complejidad, ésta debe ser entendida como una experiencia humana universal y a la vez particular a cada cultura. Pueblos de distinto origen étnico han desarrollado diferentes percepciones sobre buena y mala salud y una heterogeneidad de dolencias, síntomas 
y causas asociadas a una enfermedad. Para enfrentarlos se generan complejos sistemas de tratamiento, sanación y cura, con técnicas y roles culturalmente construidos que responden a un entorno social determinado, cuya coherencia dependerá de la sociedad en donde ha sido concebido. Estos conceptos son mecanismos explicativos de las personas, pero que podrían generar dificultad con otros actores de la salud al tener concepciones, costumbres y valores distintos (17). Es el caso de la provincia de Arauco en Chile donde coexisten dos perspectivas de la salud en un mismo territorio, la occidental a través de la red pública de salud, y la ancestral propia de la etnia mapuche (17).

El pueblo mapuche es el pueblo originario más numeroso de Chile. En la actualidad representa el 9.9\% del total de la población nacional y un $79,8 \%$ de la población indígena del país (18), concentrándose mayoritariamente en las regiones del centro-sur de Chile.

Es importante diferenciar entre el mapuche prehispano y el contemporáneo, ya que el mapuche actual presenta un importante mestizaje hispano-mapuche que es el que caracteriza a los chilenos, donde las diferencias estarían focalizadas en el terreno de la subjetividad. No obstante observarse avanzados procesos de aculturación en la población mapuche todavía es posible encontrar manifestaciones del lenguaje propio, ceremonias de índole religiosa y curativas (19).

La salud mapuche es concebida como equilibrio y armonía integral, y su transgresión se denomina enfermedad o kutran. Las mapuche kutran son enfermedades propias del mundo mapuche. Son de índole mágico-religioso y deben ser tratadas por el propio sistema médico con agentes terapéuticos indígenas y plantas medicinales. Por otro lado están las enfermedades surgidas de la occidentalización del estilo de vida, las winka kutran, y deben ser tratadas a la manera occidental (20).

Para Citarella (20) el contacto interét- nico ha llevado a que hoy se traslapen estas categorías de enfermedades originalmente excluyentes. A mayor contacto, mayor heterogeneidad de saberes y prácticas y mayor aculturación. Una enfermedad puede estar tanto en categoría mapuche como occidental, y puede ser recategorizada en el curso de la enfermedad. Es el enfermo y su familia quien toma decisiones acorde a lo que se cree causa la enfermedad, y la curación dependerá de si adscribe como mapuche u occidental.

En Chile pocas investigaciones han dado cuenta de la relación entre etnia y adherencia (21-24), no obstante evidenciar una barrera cultural en la adherencia a terapia antihipertensiva $(21,23,24)$. Al respecto, se ha planteado que debido a constructos culturales y étnicos de la población mapuche, las enfermedades crónicas resultan difíciles de definir porque confluyen en un sistema de creencias distinto al biomédico, lo que se expresa en problemas de cumplimiento de terapia farmacológica y dietoterapia, ya que los síntomas y tratamientos contradicen la cosmovisión cultural. Se percibe el tratamiento contrapuesto a la alimentación tradicional y disociativo de la historia y espiritualidad mapuche.

Bermedo (24) concluyó que el sistema cultural mapuche lleva a que los usuarios indígenas complementen, alternen o sustituyan la medicina occidental por la mapuche, constatando que las enfermedades cardiovasculares no son parte de la matriz cultural de esta etnia, generándose dificultades en la adherencia al tratamiento biomédico.

Por otra parte, para Ibacache et al. (25) el mundo mapuche ha cambiado su estilo de vida de uno ancestral a uno más occidentalizado. Esto ha tenido por consecuencia un alejamiento de las tradiciones y una pérdida de los factores protectores de la salud, donde la medicalización, la falta de información para la salud y la pérdida de la identidad han contribuido a un perfil más agresor y de mayor impacto de las enfermedades occidentales. 
Investigaciones señalan que el mapuche opta por acudir a la posta de salud en primer término, confía en los procedimientos diagnósticos y ve importante hacerse exámenes y consultar al médico ante una enfermedad, pero ignoran sus conceptos y clasificaciones (25).

No existen investigaciones que den cuenta de cómo vivencian la hipertensión y su tratamiento los hipertensos mapuche. Por tanto la presente investigación aporta en esclarecer cómo vivencia su condición de salud y tratamiento el mapuche hipertenso, con el objetivo de favorecer la implementación de un enfoque que facilite la adherencia y un mayor control sobre este problema de salud de primer orden.

\section{MATERIAL Y MÉTODO}

Se realizó un estudio de tipo cualitativo y con enfoque fenomenológico para explorar la vivencia de la hipertensión y adherencia a tratamiento en mapuche usuarios de un programa de salud cardiovascular de atención primaria.

Participaron personas mapuche diagnosticadas como hipertensas residentes en comunidades indígenas de sectores rurales de la provincia de Arauco. Para la condición de mapuche el participante debía tener al menos un apellido mapuche, en correspondencia al criterio de pertenencia indígena de la Ley Indígena No 19.253 y que se reconociera como tal. Para el criterio de hipertenso(a), la persona debía contar con el diagnóstico en su ficha clínica. Se consideró hipertenso adherente a aquel que registrara asistencia a su última citación a control; y como no adherente a quien, estando adscrito al programa, no asiste a control ni solicita hora de atención durante al menos los últimos seis meses.

La muestra fue seleccionada mediante muestreo intencionado por criterio hasta cumplir la condición de saturación de in- formación. Quedó conformada por 12 participantes entre 26 y 90 años de edad. Seis de ellos eran personas hipertensas adherentes y seis no adherentes, todas residentes de comunidades rurales de la zona de Arauco.

La técnica de recolección de información fue una entrevista en profundidad sometida a evaluación previa mediante entrevista cognitiva (26). Consistió en 12 preguntas que exploraron el conocimiento, significado y vivencia personal de la hipertensión y su tratamiento. El análisis de datos fue realizado mediante análisis temático (27). La unidad analizada fue el párrafo, donde se identificaron las unidades de significado emergentes para establecer categorías comunes en tres fases: identificación de las unidades de análisis, generación de categorías emergentes y elaboración del esquema de codificación.

El acceso a los participantes se concretó a través de los paramédicos de posta, de acuerdo a la accesibilidad y disposición para ser parte de la investigación. Las entrevistas fueron realizadas por el investigador principal y se desarrollaron en el centro de salud, en los domicilios de los participantes y en algunos lugares de trabajo en la misma comunidad indígena. Fueron registradas en su totalidad mediante grabación de audio y luego transcritas fielmente.

La investigación fue aprobada por el Comité de Ética Médica del Servicio de Salud Arauco, corresponde a una publicación original y la fiabilidad de los hallazgos fue resguardada mediante la revisión permanente de los criterios de credibilidad, transferibilidad y confirmabilidad (28). Se respetó la fiel transcripción de las entrevistas, se ubicó el marco metodológico como resguardo permanente, y se trianguló el análisis de la información con el equipo investigador. La participación anónima y voluntaria de los entrevistados fue certificada mediante consentimiento informado, en base al resguardo a los participantes de investigaciones de acuerdo a los principios propuestos por Emanuel (29). 
Para afrontar el posible riesgo previsto como consecuencia de las opiniones vertidas en las entrevistas, se resguardó la confidencialidad reemplazando los nombres por códigos en la base de datos. Para ser culturalmente pertinente se consultó periódicamente al Asesor Cultural Mapuche del servicio de salud correspondiente.

\section{RESULTADOS}

A continuación se presentan los resultados organizados en: experiencia de la enfermedad, experiencia del tratamiento y obstaculizadores y facilitadores de la adherencia.

\section{Experiencia de la enfermedad}

La enfermedad hipertensiva es experimentada con desconocimiento, no se comprende como concepto, se identifica parcializadamente como dolor de cabeza o mareos y con pocas referencias a su fisiología:

Sentía así parece que se iba a acabar el mundo, de repente me venía...parece un dolor adentro, como que parece que todo el mundo se le viene encima a uno... "parece que la cabeza adentro se me revuelve todo $y$ siento un pito (mujer, 60 años, adherente).

...ni siquiera la puedo nombrar porque es una palabra desconocida para mí (hombre, 55 años, no adherente).

La causa de la enfermedad se atribuye al carácter dañino de la alimentación occidental y se desconoce su existencia en la cosmovisión mapuche. La información diagnóstica se consideró insuficiente y en general no afectó la vida diaria:

Porque yo pienso que es una enfermedad que no está en nosotros, que no estaba, es una enfermedad que por lo mismo no la asumimos, por el hecho de que antes nosotros sufríamos otras cosas otras enfermedades, y que es una enfermedad para nosotros, por decirlo así, que no es de los mapuche... todas las enfermedades que existen hoy dia vienen de afuera (hombre, 58 años, adherente).

Si, más enfermedad de winka. Pero a algunos peñi también se le está ahora desarrollando hipertensión...tengo conocimiento que los winka viven con diabetes, y en el mapuche no hay diabético... (hombre, 90 años, adherente).

Particularmente se relacionó la hipertensión con el consumo excesivo de sal o como consecuencia de pensar mucho o de las preocupaciones:

Yo creo sinceramente que nace por los problemas de consumo. Consumimos mucha sal (mujer, 68 años, no adherente).

Será porque muchos pensamientos po. La mente trabaja mucho el celebro, el corazón. Yo entiendo así po que puede ser eso. Mucho pensamiento el celebro le duele a uno, pensar muchas cosas... (mujer, 83 años, adherente).

En un caso se identificó como de naturaleza contagiosa:

Puede que por resuellos, alguna cosa son pegativos esto... como que es pegativo algunas enfermedades que le viene uno que no conoce no tiene la ciencia ni el conocimiento de estas cosas, no puede decir (hombre, 90 años, adherente).

La experiencia emocional frente a la enfermedad es de rabia, sufrimiento y desesperación por la recurrencia de los síntomas.

\section{Experiencia del tratamiento}

La primera opción de tratamiento es farmacológico, pero se percibe con desconfianza, de efecto parcial, dañino y difícil habituación. Es ingerido sólo como calmante para 
malestares agudos, pero de efectividad disminuida si su uso es permanente:

Yo digo que son calmantes nada más. Son calmantes para el momento no más, no se introducen como estos remedios que uno toma de campo que esos están siempre ahí (hombre, 55 años, no adherente).

Los hábitos alimentarios recomendados se perciben restrictivos, poco acordes a la realidad de la comunidad mapuche y difíciles de modificar. Existe un rechazo a disminuir algunos alimentos ya que son de fácil acceso, hay dificultad para reducir el consumo de sal por rechazo al sabor insípido, y rechazo a la dieta occidental por percibirse de efecto nocivo:

Usted no tiene que tomar esto, usted no tiene que hacer esto, tiene que hacer esto otro, o a usted se está enfermando de esto, le pueda pasar esto, le puede pasar esto otro, como que lo están, entonces al final las personas sobre todo en la población mapuche, dice: yo si tengo que morir, muero no más. No me tomo los medicamentos (mujer, 68 años, no adherente).

Harina tostada, los porotos, las arvejas, esa era la comida normal, yuyo, todo eso era la comida de antes. Osea, que nosotros la misma comida que nos dan los nutricionistas esa misma comida nos están enfermando, porque tenemos que comer poquito de arroz y el arroz trae química, los tallarines igual. Y en cambio aquí comemos la comida normal, los viejitos antes no se enfermaban (mujer, 40 años, adherente).

En todos los casos el tratamiento fue complementado con especies vegetales, ya que se tiene buenas expectativas de su efecto para regular la presión, eliminar los mareos y elevar el bienestar general:

Cuando no tengo el remedio se me acaba o no tengo, mastico una hojita de Guayo...masticai una hojita de Guayo y la presión se te baja (hombre, 58 años, adherente).
Se duda del efecto del tratamiento, hay más confianza en las plantas que en el medicamento y se ingieren ante síntomas agudos. Cada participante modifica su tratamiento de acuerdo al propio sentido personal:

Cuando hay necesidad de tomar remedios tomo remedios, cuando no, pasan días y días en que no tomo ni una cosa... cuando tenía eso preparaba Limón con Natre, hee...masticaba hojas (hombre, 90 años, adherente).

Yo no he seguido mucho las indicaciones, sino que hago lo mismo y cuando se me sube la presión me tomo el remedio no más. Bueno yo igual también me recomiendo solo al final (hombre, 58 años, adherente).

La asistencia a controles se evalúa positivamente ya que ayuda a atenuar los síntomas, pero existe disconformidad y una crítica transversal a la calidad de la atención. Los profesionales se perciben con poca dedicación al paciente, de baja confidencialidad, que entregan instrucciones e información poco clara e insuficiente, y carente de reconocimiento al esfuerzo del paciente por seguir el tratamiento:

Las personas que hacen el trabajo encuentro que no lo hacen bien, no lo hacen así como dedicado...llegan y encajan las cuestiones y eso no me gusta a mí, y yo siento rechazo por eso (hombre, 55 años, no adherente).

La consulta a agentes de salud mapuche por esta problemática fue escasa, y no se mencionaron métodos diagnósticos ni categorías mapuche para la hipertensión y su tratamiento.

\section{Facilitadores y obstaculizadores de la adhe- rencia}

Entre los elementos identificados como facilitadores de la adherencia está contar con familia y redes de apoyo como compañía para 
primera consulta en la posta, para asistir a controles, o mediar hábitos alimenticios en el hogar. Se mencionó recurrentemente la participación de los hijos en la disminución de la ingesta de sal:

Tengo que ir con mi hija (a la posta), sola tampoco, sola no puedo ir.

Si po, me cuidan. Cuando yo me paso de la raya me retan (risas). Mi hija la que me cocina, ella ya aprendió a hacer comida sin sal (mujer, 88 años, no adherente).

Entre los obstaculizadores se mencionó el cambio de régimen de citación, frecuente en un inicio a esporádico para los controles, y lo aburrido de la espera por atención:

Si cuando antes recién tuve esta enfermedad que tengo me colocaban inyecciones y remedio permanentes. Pero ahora no, ahora voy cada tres meses a colocarme las inyecciones, al mes después cuando se me terminan los remedios me toman la presión..., he preguntado cómo están los exámenes y me dicen no, usted está re bien (hombre, 90 años, adherente).

Así también se deja de asistir cuando no se percibe efecto beneficioso de los medicamentos y el alivio de síntomas agudos es comprendido como ausencia de enfermedad:

Pero hallé que la tableta no me hacían (sic) nada y por eso dejé de ir a la posta (mujer, 88 años, no adherente).

Pero ahora no, ahora me toman la presión y no tengo presión (hombre, 90 años, adherente).

La atención poco dedicada y no centrada en que el paciente comprenda su problemática surge como obstaculizador de la adherencia.

La familia destaca en la regulación del autocuidado, la ingesta de sal, en el acompañamiento durante el tratamiento y la orientación para la ingesta de infusiones. La fuente de conocimiento para el uso de plantas medicinales es el uso tradicional en la familia y comunidad indígenas:

Pero mi hijo me dice mamá es un bien para usted, usted tiene que cuidarse, usted no tiene que comer sal, ¿usted sabe que es la sal? Le puede causar muchas cosas, ¿mi papá por qué murió?, por lo mismo, por los riñones. Usted no tiene que hacer lo mismo (mujer, 60 años, adherente).

Si bien todos los participantes fueron críticos con la calidad de la atención y utilizaron infusiones de plantas para controlar la presión, la condición que diferencia a adherentes y no adherentes es la carencia en estos últimos de la familia como apoyo en el curso del tratamiento.

\section{DISCUSIÓN Y CONCLUSIÓN}

Los hallazgos dan cuenta del desconocimiento y falta de comprensión en personas hipertensas mapuche sobre el significado y mecánica de la enfermedad hipertensiva como problema de salud. La posta de salud es la principal fuente de consulta, y coincidentemente con otros estudios el motivo de consulta hace referencia a síntomas fisiológicos y problemas occidentales de salud (25).

La causa de la enfermedad se atribuyó al carácter dañino de la alimentación moderna, resultados similares a otros estudios (24). La vivencia de la hipertensión se caracterizó por sentimientos de rabia, impotencia y molestia por la recurrencia de los síntomas en primer término. No es percibida como enfermedad crónica y no se identifican sus efectos en la rutina habitual, probablemente relacionado con la baja percepción de riesgo y el desconocimiento de este problema de salud como condición crónica.

Todos los entrevistados señalaron acudir a un centro de salud como primera opción 
de atención y tratamiento, lo que concuerda con la definición del problema de salud desde una dimensión occidental (24).

El tratamiento más practicado es el farmacológico aunque se percibe nocivo y con desconfianza, $y$ al igual que otras investigaciones con la misma población (21-24), se perciben dificultades en la adherencia a dietoterapia y terapia farmacológica para los problemas hipertensivos.

El presente estudio evidencia el importante rol de los hijos para incentivar la consulta y la implementación de los cambios alimentarios prescritos. Pudieran ser aspectos importantes el acompañamiento y diálogo que la familia mapuche brinda en la mantención del tratamiento, ya que es una característica que diferenció a adherentes de no adherentes. $\mathrm{Al}$ igual que en investigaciones previas $(16,24)$ son los hijos y el grupo familiar quienes influyen directamente en el curso terapéutico.

La familia extendida mapuche presenta condiciones que facilitan la adherencia, como mayor cohesión, expresión de afectos y apoyo emocional que facilita una mayor adaptabilidad. Es por ello que posiblemente se ha identificado la cohesión, la valoración del diálogo y la transmisión del conocimiento de plantas medicinales como factores promotores de la salud radicados en la familia mapuche, aspectos también observados en el presente trabajo.

Es probable que la dificultad para adherir se relacione con la disponibilidad de contención y apoyo emocional de la familia, paramédicos y profesionales, ante un problema de salud que no se comprende, que se vivencia con síntomas cíclicos que generan molestia y desesperanza, en el mismo sentido que lo han planteado investigadores que han abordado la relación entre usuario y equipo de salud $(1,30)$.

Estudios previos (21-24) identificaron problemas de adherencia asociados a la dietoterapia antihipertensiva. Específicamente, esta se opondría a la alimentación indígena, y estaría disociada de la cosmovisión y espiri- tualidad mapuche. La presente investigación ofrece evidencia en el mismo sentido al atribuir un carácter saludable a la alimentación mapuche, en contraposición al carácter dañino asignado a la alimentación occidental. Las referencias negativas apuntaron al procesamiento de los alimentos y la adición de "químicos", al elevado costo económico, al rechazo a los alimentos de gusto insípido, y a que no se incorpora en el tratamiento la alimentación natural.

En la misma línea de otros autores (21, 24) se constata dificultad en la adherencia al tratamiento asociada al uso de plantas naturales para potenciar el medicamento, afectando con ello la mantención periódica del tratamiento.

No hubo referencias a aspectos relacionados con las enfermedades espirituales mapuche. Los participantes no hicieron una descripción del problema de salud, su tratamiento o diagnóstico desde el sistema médico ancestral o como kutran, y se desconoció la existencia de la hipertensión en la cosmovisión originaria. Para su afrontamiento se recurrió en todos los casos a la posta de salud rural. Su descripción, atribución de causa, tratamiento y significado fue realizado siempre desde parámetros fisiológicos, biomédicos, farmacológicos y/o terapéuticos occidentales. Otros estudios también reportan una consulta orientada desde la perspectiva occidental (25).

La pérdida de los factores protectores culturales como el rol de los agentes tradicionales de medicina indígena $(17,8,25)$ puede ser un aspecto que relega a una posición de mayor vulnerabilidad a los pueblos originarios. En la presente investigación los entrevistados no consultaron a agentes de salud mapuche por este problema de salud.

Se es concordante con Citarella (20) en que las decisiones terapéuticas durante el curso del tratamiento se centran en la dimensión fisiológica de los síntomas, independientemente de la categoría a que se adscriba. Posiblemente el complemento o reemplazo del 
medicamento con plantas naturales obedez$\mathrm{ca}$ a la reevaluación que hace el enfermo y familia respecto de la manifestación recurrente de los síntomas fisiológicos que orientan el tratamiento.

Probablemente la preponderancia del tratamiento médico se deba a la occidentalización de la salud y enfermedad que requiere ser tratada desde la perspectiva biológica, por lo que posiblemente la tensión cultural que se ha descrito como matrices culturales contrapuestas en la concepción de la enfermedad, se encuentre sobredimensionada como aspecto interviniente en la adherencia al tratamiento.

En este estudio no se observa una contraposición de sistemas médicos occidental y mapuche en la vivencia y percepción de la enfermedad y su tratamiento, distinto a lo planteado por otros autores (21-24). Muy posiblemente la adherencia se vea afectada por la percepción de la calidad del trato, el apoyo familiar percibido y la percepción significativa de la educación en salud, como fuente de sentido y contención emocional ante un problema de salud percibido como ajeno y recurrente, más que en concepciones culturales contrapuestas.

La enfermedad hipertensiva se percibe con desconocimiento, falta de comprensión e impresión de carecer de información suficiente como problema crónico.

El conocimiento insuficiente, la desconfianza con el tratamiento y la insatisfacción con el trato representan barreras que dificultan la adherencia al tratamiento.

La carencia de consulta a sanadores ancestrales y la ausencia de identificación de la hipertensión como enfermedad pueden indicar un alejamiento de las tradiciones ancestrales.

La vivencia de la enfermedad y su tratamiento significada de modo coherente desde la perspectiva fisiológica, puede indicar que la tensión cultural esté sobredimensionada para explicar la no adherencia, pudiendo influir también otros aspectos como la empatía en la relación con el equipo de salud y el apoyo emocional percibido en la familia.

El apoyo emocional y una relación empática pueden incentivar la adherencia al tratamiento de este problema de salud que no se comprende de modo suficiente.

Algunas limitaciones del estudio deben ser consideradas, entre ellas las características sociodemográficas, identitarias y culturales para la transferencia a la población mapuche de otros territorios, la residencia urbana o de otras identidades o pueblos indígenas con realidades territoriales distintas, y que las conclusiones obtenidas deben ser comprendidas dentro de los límites que la presente muestra define.

La complejidad del problema en estudio también implica restricciones en su comprensión al abordarlo enfocando sólo la perspectiva de salud, ya que posiblemente otras dimensiones y procesos también estén presentes, entre ellos factores socioeconómicos, políticos y ecológicos. En la integralidad de este fenómeno, la perspectiva de salud es una explicación plausible dentro de la diversidad de dimensiones presentes.

Se deben considerar las diferencias culturales para la recolección de información, ya que pueden afectar las diferencias de lenguaje, códigos de relación, tiempo y suspicacia con que se percibe el mundo occidental. La dedicación en cultivar un clima acogedor y seguro para los entrevistados facilita el poder alcanzar los objetivos propuestos.

\section{REFERENCIAS}

1. Chobanian AV, Bakris GL, Black HR, Cushman WC, Green LA, Izzo JL, et al. Seventh report of the joint national committee on prevention, detection, evaluation, and treatment of high blood pressure. Hypertension 2003; 42(6): 1206-52.

2. Organización Mundial de la Salud (OMS). Información general sobre la hi- 
pertensión en el mundo. Una enfermedad que mata en silencio, una crisis de salud pública mundial [Internet]. Ginebra: OMS; 2013 [citado 24 jun 2017]. 40 p. Disponible en: http://apps.who.int/iris/ bitstream/handle/10665/87679/WHO_ DCO_WHD_2013.2_spa.pdf;jsessioni$\mathrm{d}=$ 167DF1B6860F434D2DF091C4CCCC7556? sequence $=1$

3. Benavides V, Jaramillo L, Rendón S, Valenzuela A, Argotty E, Mafla A. Determinantes de adherencia al tratamiento hipertensivo en adultos $\geq 35$ años de edad. Univ. Salud. 2013; 15(2): 136-49.

4. Ministerio de Salud (CL), Pontificia Universidad Católica de Chile, Universidad Alberto Hurtado. Encuesta Nacional de Salud 2009-2010 [Internet]. Santiago, Chile: Ministerio de Salud; 2009 [citado 17 sep 2017]. Disponible en: http://www. minsal.cl/portal/url/item/bcb03d7bc28b64dfe040010165012d23.pdf

5. Vidal D, Chamblas I, Zavala M, Müller R, Rodríguez M, Chávez A. Determinantes sociales en salud y estilos de vida en población adulta de Concepción, Chile. Cienc. enferm. 2014; XX(1): 61-74.

6. Ministerio de Salud (CL). Perfil epidemiológico básico de la población aymara de la Provincia de Iquique. Serie análisis de la situación de salud de los pueblos indígenas de Chile No 3 [Internet]. Santiago, Chile: Ministerio de Salud; 2007 [citado 21 dic 2017]. 66 p. Disponible en: http://www.minsal.cl/sites/default/files/ files/SERIE\%20PUBLICACIONES\%20 SI T UACI \% C $3 \% 93 \mathrm{~N} \% 20 \mathrm{DE} \% 20$ SALUD\%20N\%C2\%B0\%203\%20IQUIQUE.pdf

7. Ministerio de Salud (CL). Perfil epidemiológico básico. Pueblos Kawésqar, Yámana y Mapuche-williche, Región de Magallanes. Serie análisis de la situación de salud de los pueblos indígenas de Chile No 4 [Internet]. Santiago, Chile: Ministerio de Salud; 2009. [citado 21 dic 2017]. 63 p. Disponible en: http:// www.minsal.cl/sites/default/files/files/ SERIE\%20PUBLICACIONES\%20SITUACION\%20DE\%20SALUD\%20\%20 N\%C2\%B05\%20MAGALLANES.pdf

8. Ministerio de Salud (CL). Perfil epidemiológico básico de la población mapuche residente en la Provincia de Arauco. Serie análisis de la situación de salud de los pueblos indígenas de Chile No 7 [Internet]. Santiago, Chile: Ministerio de Salud; 2010 [citado 21 dic 2017]. 81 p. Disponible en: http://www.minsal.cl/sites/ default/files/files/SERIE\%20PUBLICACIONES\%20SITUACION\%20DE\%20 SALUD $\% 20 \mathrm{~N} \% \mathrm{C} 2 \% \mathrm{~B} 07 \% 20$ ARAUCO. pdf

9. Oyarce A, Pedrero M (Programa Orígenes, mira el futuro desde tu origen). Perfil epidemiológico de la población mapuche residente en el área de cobertura del Servicio de Salud Provincia del Bío-Bío. Serie análisis de estudios de salud de los pueblos indígenas de Chile. Región del Bío-Bío No 1. Santiago, Chile: Ministerio de Salud; 2011. [citado 21 dic 2017]. 77 p. Disponible en: http://www.minsal.cl/ sites/default/files/files/Edicion\%20Perfil\%20Epidemiol $\%$ C3\%B3gico $\% 20 \mathrm{~B} \%$ C3\%A1sico\%20de\%20B\%C3\%ADo\%20 B\%C3\%ADo.pdf

10. Martin L, Grau J, Espinosa A. Marco conceptual para la evaluación y mejora de la adherencia a los tratamientos médicos en enfermedades crónicas. Rev Cub Salud Publica. 2014; 40(2): 225-35.

11. Ortiz M, Ortiz E. Psicología de la salud: una clave para comprender el fenómeno de la adherencia terapéutica. Rev Med Chile. 2007; 135(5): 647-52.

12. Ortiz G, Ortega E. Capacidad predictiva de la adherencia al tratamiento en los modelos socio-cognitivos de creencias en salud. Psicología y Salud. 2011; 21(1): 7990.

13. Sandoval D, Chacón J, Muñoz R, Henríquez $\mathrm{O}$, Koch $\mathrm{E}$, Romero $\mathrm{T}$. Influencia de factores psicosociales en la adheren- 
cia al tratamiento farmacológico antihipertensivo. Resultados de un cohorte del programa de Salud Cardiovascular de la Región Metropolitana, Chile. Rev Med Chile. 2014; 142(10): 1245-52.

14. Zurera I, Caballero M, Ruiz M. Análisis de los factores que determinan la adherencia terapéutica del paciente hipertenso. Enferm Nefrol. 2014; 17(4): 251-260.

15. Alemán J, Rueda B. Factores asociados a la vulnerabilidad emocional y la baja adherencia al tratamiento en la hipertensión esencial. Boletín de Psicología. 2014; 112: 35-52.

16. Canales S, Barra E. Autoeficacia, apoyo social y adherencia al tratamiento en adultos con diabetes mellitus tipo II. Psicología y Salud. 2014; 24(2): 167-173.

17. Pérez C, Nazar G, Cova F. Facilitadores y obstaculizadores de la implementación de la política de salud intercultural en Chile. Rev Panam Salud Publica. 2016; 39(2): 122-127.

18. Instituto Nacional de Estadísticas (CL). Segunda entrega de resultados definitivos Censo 2017 [Internet]. Santiago: Instituto Nacional de Estadísticas; 2017 [citado 30 abr 2018]. 56 p. Disponible en: http://www.censo2017.cl/wp-content/ uploads/2018/05/presentacion_de_la_ segunda_entrega_de_resultados_censo2017.pdf

19. Marimán J. Autodeterminación. Ideas políticas mapuche en el albor del siglo XXI. Santiago: Lom Ediciones; 2012. 358 p.

20. Citarella L. editor. Medicinas y culturas en la Araucanía. $2^{\text {a }}$ ed. Santiago, Chile: Editorial Sudamericana; 1995.616 p.

21. Oliva P, Narváez C. Representaciones sociales de salud y adherencia farmacológica antihipertensiva en población pehuenche. Rev Chil Salud Pública. 2009; 13(2): 67-71.

22. Oliva P, Narváez C. Cumplimiento de tratamientos farmacológicos: una aproximación cualitativa a la realidad pehuen- che. Medwave. 2013; 13(6): 1-7.

23. Oliva P, Buhring K. Problemas de adherencia a dietoterapia en pacientes hipertensos pehuenches. Rev Chil Nutr. 2011; 38(3): 285-89.

24. Bermedo S. Dimensiones y significados que adquiere el proceso salud/enfermedad/atención en usuarios mapuche-williche con diabetes mellitus e hipertensión arterial. Rev Chil Salud Pública. 2015; 19(1): 47-52.

25. Ibacache J, McFall S, Quidel J. Rume kagenmew ta az mapu. Epidemiología de la transgresión en Makewe-Pelale. Working paper series 2 [Internet]. [lugar desconocido]: Nuke mapuförlaget; 2002 [citado 21 mar 2017]. Disponible en http://www. mapuche.info/wps_pdf/Ibacache020200. PDF

26. Willis G. Cognitive interviewing: A tool for improving questionnaire design. London: Sage publications; 2005. 352 p.

27. Braun V, Clarke V. Using thematic analysis in psychology. Qual. Res. Psych. 2006; 3(2): 77-101.

28. Castillo E, Vásquez M. El rigor metodológico en la investigación cualitativa. Colomb Med. 2003; 34(3): 164-77.

29. Emanuel E. ¿Qué hace que la investigación clínica sea ética? Siete requisitos éticos. En: Lolas F, Quezada A. Pautas éticas de investigación en sujetos humanos: nuevas perspectivas [Internet]. Santiago de Chile: Programa Regional de Bioética OPS/ OMS; 2003 [citado 21 sep 2017]. p. 8590. Disponible en: https://www.paho.org/ chi/index.php?option $=$ com_docman $\&$ view $=$ download $\&$ alias $=82$ - pautas - eti cas-de-investigacion-en-sujetos-humanos-nuevas-perspectivas\&category_slu$\mathrm{g}=$ documentos-tecnicos\&Itemid $=1145$

30. Garrido J, Chacón J, Sandoval D, Muñoz R, López N, Oyarzún E et al. Control del hipertenso, un desafío no resuelto. Avances logrados en Chile mediante el Programa de Salud Cardiovascular. Rev Chil Cardiol. 2013; 32(2): 85-96. 